\title{
One Observer Universe a geometric interpretation of speed of light and mass
}

\author{
Ahmed Farag Al* \\ Department of Physics, Faculty of Science, Benha University, 13518, Egypt.
}

\begin{abstract}
In this paper, we investigate how Rindler observer measures the universe in the ADM formalism. We compute his measurements in each slice of the space-time in terms of gravitational red-shift which is a property of general covariance. In this way, we found special relativity preferred frames match with general relativity Rindler frame in ADM formalism. This may resolve the widely known incompatibility between special relativity and general relativity on how each theory sees the redshift. We found a geometric interpretation of speed of light and mass.
\end{abstract}

\section{ONE PREFERRED OBSERVER}

The absolute space and time means by definition a preferred frame, in which physics laws appear to be recognizably different from they appear in other frames. This Newtonian concept is contradicted with the assumed principle of relativity in inertial frames and the principle of covariance in general theory of relativity. However, all inertial frames are preferred over non-inertial frames in Newtonian and special relativity mechanics as they observe only cause-and-effect relations between events in closed intervals, a property that general relativity lacks as it can observe a cause-and-effect relations between internal and external events. In this paper, we use the Rindler observer and the ADM formalism to restore the compatibility between the inertial privileged frames and relative space of general relativity, at least slice by slice. It is worth noting that despite we agree with that absolute space in Newtonian gravity has no meaning, then, according to Mach principle, it is still can be obtained only under the condition that there exists in the universe only one single object, nothing more For more details on this issue, see [1, 2].

We consider spacetime foliation in ADM formalism [3, at every given moment according to canonical observers, each with its speed at that moment. These observers are related to each other by "synchronicity" 4. This approach comes with a global reference frame that stays Minkowskian along the worldline of such observer. Despite that the absolute synchronicity is not attainable either in special relativity or in general relativity, a local synchronicity is extended beyond local neighborhoods as in Ref. [4]. We Consider a black hole spacetime as a spacetime with horizon. Such horizon is necessarily observer-dependent. This requires the Hawking radiation to be with Unruh effect as in Ref. [4]. Also from the Rindler observer [5] would analyze the ADM formalism as if the shift function equal zero $N_{i}=0$. Therefore, the normal vector $\hat{n}$ to the spatial slice is proportional to the time basis $\partial_{t}$ of Rindler frame field with proportionality factor that is equal to the

\footnotetext{
*email: ahmed.ali@fsc.bu.edu.eg
}

lapse function $\alpha$. In this case the coordinate observer is moving with velocity $\left(g_{t t}\right)^{-1 / 2} e_{t}$ while the normal observer moves with velocity $\hat{n}$. The factor similar to $\gamma$ in ADM formalism is in form of $\left(1-N_{i} N^{i} / \alpha^{2}\right)^{-1 / 2}$. For the Rindler observer, the previous factor is equal to 1. However this comparison between observers is NOT a physical relativist Lorentz boost, and the frame of reference can be dragged with any speed the observer chooses. It turns out that the normal observer and the Rindler observer are the same or One Observer. They both see each other as if they are both belong to the same inertial Minkowski frame. This matches with the global reference frame described in Ref. [4].

\section{ONE OBSERVER MEASUREMENTS}

The One Observer measures the gravitational red-shift which is a property of general covariance and its relative values as the one observer moves from point to point or from slice to slice. This can be listed as below

1. general covariance measurement: by uniformly accelerated Rindler observer: The shift in energy which is the gravitational red-shift in the black hole case.

$$
z_{A}=\left(1-\frac{r_{s}}{r_{A}}\right)^{-1 / 2}-1
$$

where $r_{s}$ is Schwarzschild radius, $r_{A}$ is the distance at which red-shift is measured. Notice that we do not measure the energy exactly at the horizon as the time there freezes with respect to the observer. Rather the observer measures the escaped Hawking radiation, after considering the Unruh effect, which is entangled with the unlucky radiation that the observer does not detect as those unlucky particles fall into the horizon.

\section{Relative or local measurements:}

(a) We mean by relative or local measurements the ratio between gravitational red-shifts at different slices or two different points. The 
ratio represents the relative gravitational redshift

$$
\frac{z_{A}}{z_{R}}=\frac{\left(1-\frac{r_{s}}{r_{A}}\right)^{-1 / 2}-1}{\left(1-\frac{r_{s}}{r_{R}}\right)^{-1 / 2}-1}
$$

(b) The local measurements can be also the difference in gravitational red-shift at two different slices or two different points. The difference in gravitational red-shift is given by

$$
\Delta z=z_{A}-z_{R}=\left(1-\frac{r_{s}}{r_{A}}\right)^{-1 / 2}-\left(1-\frac{r_{s}}{r_{R}}\right)^{-1 / 2}
$$

To compute these relative or local measurements, a triangle is constructed that its vertices represent the black hole and any two points in the space as shown in Fig. (1). $K$ point refer to the black hole. $A$ and $R$ refers to any two different points or two different slices.
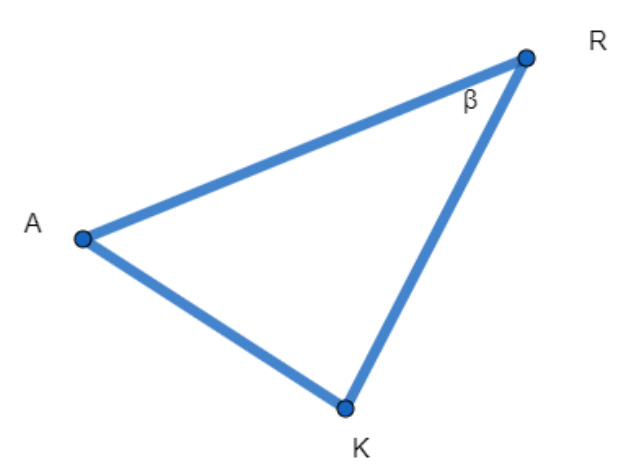

FIG. 1: The triangle of one observer measurements

Notice here this triangle follow the geodesics geometry of the considered black hole to connect the three points with each other. If $R$ and $A$ are far enough from $K$, the triangle become approximately Euclidean triangle.

\section{RELATIVE MEASUREMENTS OF GRAVITATIONAL RED-SHIFT}

We calculate the relative gravitational red-shift between $A$ and $R$ when $r_{A} \neq r_{R}$. We consider the triangle in Fig.(1). For brief symbols, We define:

$$
\begin{gathered}
A K=r_{A} \\
R K=r_{R} \\
A R=r_{A R}
\end{gathered}
$$

\section{A. weak gravitational case}

We consider first the simple case in which we set $r_{s}<<$ $r_{K}$ and $r_{s}<<r_{R}$. The gravitational red-shift for both $\mathrm{A}$ and $\mathrm{R}$ can be approximated as follows

$$
\begin{aligned}
& z_{A}=\left(1-\frac{r_{s}}{r_{A}}\right)^{-1 / 2}-1 \approx \frac{r_{s}}{2 r_{A}} \\
& z_{R}=\left(1-\frac{r_{s}}{r_{R}}\right)^{-1 / 2}-1 \approx \frac{r_{s}}{2 r_{R}} .
\end{aligned}
$$

We compute the relative gravitational red-shift using $\mathrm{Eq}(2)$. We express it in terms of all lengths measured at $R$ including the distance between $A$ and $R\left(r_{A R}\right)$.

$$
\frac{z_{R}}{z_{A}}=\frac{r_{A}}{\sqrt{r_{A}^{2}-r_{A R}^{2}+2 r_{R} r_{A R} \cos \beta}}
$$

This equation can be rewritten as follows

$$
\frac{z_{R}}{z_{A}}=\frac{1}{\sqrt{1-\frac{r_{A R}^{2}}{r_{A}^{2}}+2 \frac{r_{R} r_{A R}}{r_{A}^{2}} \cos \beta}}=\delta
$$

where $\delta=1 / \sqrt{1-\frac{r_{A R}^{2}}{r_{A}^{2}}+2 \frac{r_{R} r_{A R}}{r_{A}^{2}} \cos \beta}$. Notice the value of $\beta$ can be $0 \leq \beta \leq \pi / 2$. This equation represents the relative gravitational-red-shift between two points $A$ and $R$ in a weak gravitational field.

For the case $\beta=\pi / 2$. The relative gravitational redshift will be given by

$$
\frac{z_{R}}{z_{A}}=\frac{1}{\sqrt{1-\left(\frac{r_{A R}}{r_{A}}\right)^{2}}}
$$

This relation says the relative gravitational red-shift is always bounded by the distance of the point which is distant from the black hole which in this case the point A. This form an invariant boundary on the measured relative gravitational-red-shift which is determined by the distance from the black hole. Notice the similarity between $\delta$ factor in Eq. (11) and gamma factor in special relativity $\gamma=\sqrt{1-v^{2} / c^{2}}$, where $v$ is the relative velocity. This means in this special case, when $\beta=\pi / 2$, the gamma factor of special relativity emerges as a ratio between the gravitational red-shift at $A$ and red-shift at $R$ for the one observer. The one observer moves from one slice to slice, which is the reason for velocity ratios turned to be lengths ratios in this gamma factor in Eq. (11). The ratio $r_{A R}^{2} / r_{A}^{2}$ can be considered as a geometric interpretation of the ratio $v^{2} / c^{2}$ which can be realized by Rindler observer between two different slices of the space-time.

We notice here that the comparison between the factors $r_{A R}^{2} / r_{A}^{2}$ and $v^{2} / c^{2}$ make a correspondence between $r_{A R}$ and $v$ on one side and a correspondence between $r_{A}{ }^{2}$ and $c^{2}$ on the other side. It is logical that relative speed between $A$ and $R$ is related to the distance between them. But we found an interesting result for the One observer that speed of light will be varying as he moves close or far from the gravitational source or black hole. This would suggest that speed of light decreases in 
strong gravity field and increases as the observer moves far from the source. This would support the approach of time varying speed of light as a solution of cosmological puzzles that was suggested in 8 .

To understand the effect of other values of angle $\beta$ in weak gravitational field, we consider an approximation which is $r_{A R}<<r_{A}, r_{A R}<<r_{R}$. In that case, the delta factor in Eq. 10 is approximated as following

$$
\delta \approx 1-\frac{r_{R} r_{A R}}{r_{A}^{2}} \cos \beta
$$

It is found that this equation matches with the derivative of Kepler equation.

$$
\frac{d M}{d E}=1-e \cos E
$$

where $M$ is the mean anomaly, $E$ is the eccentric anomaly, and $e$ is the eccentricity. In our approximation, the eccentricity $e$ is approximately equal to $r_{R} r_{A R} / r_{A}^{2}$, and $E$ refers to the angle $\beta$. This match was expected, because we study the change in relative gravitational field in slice by slice of the space-time, and the derivative of Kepler equation represents the change of gravity in a segment by segment of the space. This once again give a geometric interpretation of Kepler equation from the relative gravitational red-shift.

Let us now focus on the general relative relation between gravitational red-shifts at any distance from the black hole, which gives the strong gravity case.

\section{B. strong gravity case}

By using Eq. (8), the relative relation between gravitational red-shift at $A$ and $R$ are as following:

$$
\frac{z_{R}}{z_{A}}=\frac{\left(1-\frac{r_{s}}{r_{R}}\right)^{-1 / 2}-1}{\left(1-\frac{r_{s}}{r_{A}}\right)^{-1 / 2}-1}
$$

This relation can be computed for any two points, and it gives a wide spectrum of measurements of relative gravitational red-shifts in strong gravity field. Notice here that the distance between $A$ and $R$ through the triangle relation between $A, R$ and the black hole $K$. In strong gravity field, the triangle will not be perfectly Euclidean but can be computed for every kind of measurement by knowing the length of this triangle and apply it in Eq. 14.

\section{DIFFERENCE IN GRAVITATIONAL RED-SHIFT}

In this section, we compute how Rindler observer measures the difference in gravitational red-shift. Let us make our computations in the case of weak gravitational red-shift

$$
\Delta z=z_{R}-z_{A}=\frac{r_{s}}{2 r_{R}}-\frac{r_{s}}{2 r_{A}}
$$

We assume an approximation as folliwng $r_{A}=r_{R}+x$, where $x<<r_{A}$ and $x<<r_{R}$.

In that case, Eq. (15) will be rewritten as follows. We use the value of Schwarzschild radius $r_{s}=2 G M / c^{2}$

$$
\Delta z c^{2}=G M \frac{x}{r_{R}^{2}}
$$

where $G$, is the gravitational constant, $M$, is the black hole mass and $c$ is the speed of light. We find that Eq. (16) can be arranged to take the following form

$$
\Delta z M c^{2}==\Delta M c^{2}=G M^{2} \frac{x}{r_{R}^{2}}
$$

where $\Delta M=\Delta z M . \Delta M$ represents the emergence of mass as the observer moves between two different slices. This would give a geometric representation for relativistic relation $m c^{2}$ in terms of the distances of the different points to the black hole. We want to understand the physical meaning of the factor $G M^{2}$ in r.h.s of Eq. (17). When we look at Bekenstein-Hawking entropy equation

$$
S_{B H}=\frac{c^{3} A}{4 G \hbar}=\frac{4 \pi}{c \hbar} G M^{2}
$$

where $A=16 \pi\left(G M / c^{2}\right)^{2}$ stands for surface area of a black hole. We found that the factor $G M^{2}$ in r.h.s of Eq. (17) between any two different points can be expressed in terms of black hole entropy as follows

$$
\Delta z M c^{2}=\Delta M c^{2}=\frac{c \hbar}{4 \pi} \frac{x}{r_{R}^{2}} S_{B H}
$$

This relation can be rewritten as follows

$$
\Delta M=2.79927514 \times 10^{-44} \times \frac{x}{r_{R}^{2}} S_{B H}
$$

This relation may establish a connection between the concept of measured mass by the One observer as he moves between two different slices and and black hole entropy when the one observer measures the mass in a slice by slice. Note the relation that connect the measured mass with the entropy is a geometric relation that depends on the factor $\left(x / r_{R}^{2}\right)$.

We notice that the difference in gravitational red-shift implies an emergence of mass. It is experimentally proved that the difference in gravitational potential has an effect on the apparent weight of the $14.4-\mathrm{keV}$ ray of Iron $(\mathrm{Fe}) 6$, 7]. This may be an experimental support for the derived relation that connect the difference in gravitational redshift and emergence of mass in this section. 


\section{LOCALITY FOR THE ONE OBSERVER}

We notice that the one observer measures relative measurements in terms of the ratio of gravitational red-shift at two different points and in terms of the difference in gravitational red-shift between two different points. These two kind of local measurements vanishes only when the One observer moves through slices on a perfect sphere around the black hole, in which he measures no relative gravitational red shift and the difference will be zero. This means the One observer only perform local measurements as far as he moves between different slices at different distances from the black hole. For slices on the perfect spheres, no local measurements can be computed. He only can measure the gravitational red-shift which is a property of general covariance at each perfect sphere around the black hole. This implies that locality is related to measuring relative gravitational red-shift. This may explain why special relativity inertial frames holds only locally .

\section{ONE OBSERVER AT $r_{A}=r_{R}$}

When the one observer moves through slices on the same perfect sphere around the black hole, it gives no relative gravitational-red-shift. In that case, we find that

$$
z_{A}=z_{R}
$$

In that specific state, there is no any relative gravitational red-shift between the two points $A$ and $R$. In this case, patterns of spheres are created through. It gives only an absolute measurement of gravitational red-shift over that sphere. These spheres does have internal symmetry that is independent from its position to the black hole. Note here that the difference in gravitational redshift on the same sphere is equal to zero and therefore the mass, which implies that $\Delta z=0$ and $\Delta M=\Delta z M=0$ which explains why the light is mass-less from geometric interpretation.

\section{TIME AS A CONTINUUM CHANGE}

The continuum of relative measurements of relative gravitational red-shift implies relative time counts only when the two points $A$ and $R$ exist at "two" different distances from the black hole. relative time seems to be a rule of "two". The observer can measure a change in the space while dragging the frame from a slice to another. Those dragging process is what creates the feeling of causality between events in the former slice and those in the subsequent slice.

\section{CONCLUSIONS}

In this paper, we investigate how Rindler observer in ADM formalism make measurements in the slices of space-time. We found that the Rindler observer in ADM formalism restores the compatibility between the inertial privileged frames and relative space of general relativity, at least slice by slice. We determined the relative or local measurements that One observer could make in each slice of the space-time, and we found a geometric interpretation of speed of light and the mass. Besides, we found that when the one observer moves through slices on a perfect sphere around the black hole, the local measurements vanishes which implies that locality is related to relative gravitational red-shift.

Acknowledgment The author is deeply grateful for enlightening discussions with Hassan Alshal. This work is supported by the quantum gravity research grant, Los Angeles, California.
[1] Ciufolini, Ignazio, and John Archibald Wheeler. Gravitation and inertia. Princeton university press, 1995.

[2] Norton, John D. Reports on progress in physics 56, no. 7: 791.(1993); Norton, John D. "Einstein, Nordstrom, and the Early Demise of Scalar, Lorentz Covariant Theories of Gravitation." The genesis of general relativity. Springer, Dordrecht, 2007. 1337-1411.

[3] R. L. Arnowitt, S. Deser and C. W. Misner, Gen. Rel. Grav. 40, 1997 (2008) doi:10.1007/s10714-008-0661-1 [grqc/0405109].

[4] M. Lachieze-Rey, Astron. Astrophys. 376, 17 (2001). [gr- qc/0107010].

[5] W. Rindler, Am. J. Phys. 34, 1174 (1966).

Annalen Phys. 35, 898 (1911)

[6] R. V. Pound and G. A. Rebka, Jr., Phys. Rev. Lett. 4, 337 (1960). doi:10.1103/PhysRevLett.4.337

[7] R. V. Pound and J. L. Snider, Phys. Rev. Lett. 13, 539 (1964). doi:10.1103/PhysRevLett.13.539

[8] A. Albrecht and J. Magueijo, Phys. Rev. D 59, 043516 (1999) doi:10.1103/PhysRevD.59.043516 [astro$\mathrm{ph} / 9811018]$. 